\title{
P Wave Analysis in Patients with Sarcoidosis
}

\author{
Elias Gialafos ${ }^{1,2 *}$, Elias Perros ${ }^{3 * \#}$, Aggeliki Rapti ${ }^{4}$, Theodore G. Papaioannou ${ }^{5}$, Vassilios Kouranos ${ }^{4}$, \\ Ioannis Moyssakis ${ }^{4}$, Konstantina Aggeli ${ }^{5}$, Georgios Dimopoulos ${ }^{6}$, Charalambos Kostopoulos ${ }^{3}$, \\ Eleftherios Stamboulis ${ }^{1}$, John Gialafos ${ }^{5}$, Christodoulos Stefanadis ${ }^{5}$, Nikolaos Koulouris ${ }^{2}$, \\ Myron Mavrikakis $^{3}$
} ${ }^{1}$ Department of Neurology, University of Athens, Athens, Greece; ${ }^{2}$ Department of Pulmonology, University of Athens, Athens,
Greece; ${ }^{3}$ Department of Clinical Therapeutics, University of Athens, Athens, Greece; ${ }^{4}$ Outpatient Department of Sarcoidosis, Chest
Disease Hospital "Sotiria", Athens, Greece; ${ }^{5}$ Department of Cardiology, University of Athens, Athens, Greece; ${ }^{6}$ Department of Criti-
cal Care Medicine, University of Athens, Athens, Greece.

Email: " eliasperros@yahoo.com

Received June $12^{\text {th }}, 2013$; revised July $16^{\text {th }}, 2013$; accepted August $5^{\text {th }}, 2013$

Copyright (C) 2013 Elias Gialafos et al. This is an open access article distributed under the Creative Commons Attribution License, which permits unrestricted use, distribution, and reproduction in any medium, provided the original work is properly cited.

\begin{abstract}
Introduction: Atrial arrhythmias in patients with sarcoidosis (Sar) are not unusual and can occur due to either atrial myocardial fibrosis and/or due to autonomic nervous system imbalance. Electrocardiographic markers (ECG), like maximum and minimum $\mathrm{P}$ wave duration and $\mathrm{P}$ wave dispersion $\left\{\mathrm{P}_{\mathrm{dis}}=\mathrm{P}_{\max }-\mathrm{P}_{\min }\right\}$ reflect atrial depolarization inhomogeneity and can indicate patients prone to develop atrial arrhythmias while standard deviation of RR interval (SDNN) is an index of heart rate variability, reflecting autonomic nervous system (ANS) activity. Methods: 90 patients with sarcoidosis (41 males/49 females) enrolled in this multicenter prospective study underwent digital electrocardiography, echocardiography and pulmonary function tests (PFTs). Diastolic and systolic indices of right and left ventricle were measured echocardiographically including Doppler parameters while $\mathrm{P}_{\max }, \mathrm{P}_{\min }, \mathrm{P}_{\mathrm{dis}}$ and SDNN were measured in a 5minute duration digital electrocardiogram. All consecutive patients were compared to 65 healthy volunteers $(30$ males/35 females). Results: Although heart rate and the echocardiographic indices were similar among the two groups, the electrocardiographic indices were significantly prolonged in the patient group compared to controls. Maximum $P$ wave duration was correlated with SDNN $(\mathrm{p}<0.05, \mathrm{r}=-0.272)$ and the age of the patients $(\mathrm{p}<0.05, \mathrm{r}=0.219)$ while $\mathrm{P}_{\text {dis }}$ was correlated with SDNN $(\mathrm{p}<0.001, \mathrm{r}=0.350)$ and the heart rate $(\mathrm{p}<0.005, \mathrm{r}=0.323)$. Multivariate analysis showed that $\mathrm{P}_{\max }$ and $\mathrm{P}_{\text {dis }}$ were independently correlated with SDNN. Conclusion: $\mathrm{P}$ wave dispersion is significantly increased in patients with systemic sarcoidosis compared to healthy persons while maximum $\mathrm{P}$ wave duration and $\mathrm{P}$ wave dispersion are negatively correlated with the standard deviation of RR, an index of heart rate variability implying imbalance of ANS function. Further studies are needed for the clarification of the significance of this correlation.
\end{abstract}

Keywords: P Wave Analysis; Systemic Sarcoidosis; Autonomic Nervous System

\section{Introduction}

Sarcoidosis (Sar) is a systemic granulomatous disease of unknown etiology characterized by variable clinical manifestations and an unpredictable course [1]. Myocardial involvement is considered as a major contributor to mortality in patients with sarcoidosis [2-4]. Cardiac manifestations range from an incidentally identified, benign condition to fatal cardiomyopathy causing cardiac arrhythmias and sudden cardiac death $[5,6]$. Conduction abnormalities that range from first degree AV block to com-

\footnotetext{
*Elias Gialafos and Elias Perros have contributed equally to this work.

${ }^{\#}$ Corresponding author.
}

plete heart block are the most common clinical manifestations of cardiac involvement, with ventricular and atrial arrhythmias being less frequent. Atrial arrhythmias have an incidence of up to $19 \%$ with atrial tachycardia, atrial flutter and atrial fibrillation being more common.

Simple electrocardiographic markers like maximum $\left(\mathrm{P}_{\max }\right)$, minimum $\left(\mathrm{P}_{\min }\right)$ and dispersion $\left(\mathrm{P}_{\mathrm{dis}}\right)$ of $\mathrm{P}$ wave are well known electrophysiological characteristics of atria prone to fibrillate and are associated with inhomogeneous and discontinuous propagation of sinus impulses $[7,8]$. $\mathrm{P}$ wave indices have been studied in several diseases such as hypertension, aortic stenosis, dilated cardiomyopathy and ischemia with common conclusion that 
prolongation of $\mathrm{P}_{\max }$ and $\mathrm{P}$ wave dispersion signifies increased risk for the development of atrial fibrillation [8-12]. Standard deviation of RR interval (SDNN) is an index of heart rate variability and is considered as a possible marker of autonomic nervous system imbalance [13].

The incidence of symptomatic cardiac involvement in sarcoidosis is 5\%, but autopsy material has shown at least $20 \%-27 \%$ of the patients $[14,15]$. Atrial arrhythmias are considered as one of the clinical criteria for the diagnosis of cardiac sarcoidosis according to the Japanese ministry criteria [16]. As a result, the identification of patients prone to develop this kind of arrhythmia might play a significant role in the treatment and alter the course of the disease, since subclinical cardiac involvement may play an important role in the disease's progression and prognosis.

This study was conducted in order to identify these patients with the use of $\mathrm{P}$ wave analysis.

\section{Patients and Methods}

\subsection{Study Population}

An observational case-control study was conducted with patients from three university hospitals with biopsy proven sarcoidosis between October 2002 and June 2004. Presence of non caseating granulomas in the transbrochial biopsy, lymph node or skin biopsies confirmed the diagnosis of sarcoidosis. The possibility of infection, environmental factors or hypersensitivity reaction to medication causing granulomatous inflammation had been eliminated. Treatment with systemic glucocorticoids was not an exclusion criterion. Exclusion criteria were the presence of chronic obstructive lung disease, presence of arrhythmias, known coronary artery disease or structural heart disease, systemic hypertension, diabetes mellitus, pericarditis, pregnancy, alcoholism and the presence of a previously implanted pacemaker. All consecutive patients were compared to a control group of healthy asymptomatic volunteers with no comorbidities and any past or present evidence of heart and/or lung disease. None of the patients or control subjects was receiving any cardiac medication.

All consecutive patients underwent clinical assessment, including determination of serum levels of angiotensinconverting enzyme, 12-lead ECGs, radiologic chest stage by radiography and transthoracic echocardiograms. Chest radiographs were assessed to determine disease stage using standard radiographic staging for sarcoidosis according to the Scadding criteria: I, bilateral hilar lymphadenopathy (BHL) with normal lung parenchyma; II, BHL and parenchymal infiltration; III bilateral infiltrates without BHL and Stage IV pulmonary fibrosis/fibrocystic parenchymal involvement [16]. Serum angiotensin converting enzyme [SACE] as well as brain natriuretic peptide (BNP, Triage, ROCHE), an estimator of diastolic function in the left ventricle, were measured. High SACE activity was defined as concentration higher than $55 \mathrm{U} / \mathrm{L}$ and was considered to reflect disease activity $[18,19]$, while BNP higher than $100 \mathrm{pg} / \mathrm{dl}$ implied diastolic heart failure (Table 1). Control subjects underwent complete echocardiographic and electrocardiographic study. The study protocol was approved by the institutional ethics committee and informed consent was obtained from all the study subjects.

\subsection{Pulmonary Evaluation}

Pulmonary function tests (PFTs) including Forced Expiratory Volume at $1 \mathrm{sec}$ (FEV1), Forced Vital Capacity (FVC), the ratio of FEV1/FVC and the Total Lung Capacity (TLC) were performed with a body box plethysmograph while Diffusing Capacity for Carbon Monoxide (DLCO) was measured by the single breath method. Values were expressed as a percentage of those predicted.

\subsection{Standard Echocardiography}

An echocardiographic study was performed for all participants in the same echo lab with the patients in partial left decubitus position by an expert sonographer using a commercially available ultrasonic device (Hewlett-Packard, Sonos 5500, Andover, Massachusetts). All M-mode, two dimensional and Doppler images were recorded with a S3 transducer. Two-dimensional guided M-mode quantitative left ventricular analysis was performed in the parasternal short-axis view, according to the recommendations of the American Society of Echocardiography. [20] Left atrial (LA), diastolic (LVEDD) and systolic (LVESD) ventricular dimensions were measured. Left ventricular Ejection Fraction (EF) was determined by the biplane Simpson's method, [21] whereas LV mass by the Penn Conversion Formula. [22] Pulsed Doppler recordings were obtained during transmitral and transtricuspidal flow and the following parameters were measured: maximal velocity of early diastolic filling $\left(E_{m}\right)$ or $\left(E_{t}\right)$, late diastolic filling $\left(\mathrm{A}_{\mathrm{m}}\right)$ or $\left(\mathrm{A}_{\mathrm{t}}\right)$ and the ratio of early to late diastolic filling velocities $\left(E / A_{m}\right.$ or $\left.E / A_{t}\right)$ in mitral or tricuspid valve, respectively. Furthermore, Deceleration Time (DT) and Isovolumic Relaxation Time (IVRT) of mitral and tricuspid valves were measured.

\subsection{2-Lead Surface ECG}

In all participants, a 12 lead digital ECG was recorded in the supine resting position using a computer-based ECG system (CardioControl NV, the Netherlands). The 12 leads of ECG were recorded simultaneously at a sampling rate of $1200 \mathrm{~Hz}$ for 5 minutes. During each re- 
Table 1. Baseline demographic and clinical patients' and controls' characteristics, ultrasound and electrocardiographic parameters among groups of patients with and without therapy and controls.

\begin{tabular}{|c|c|c|c|c|}
\hline Patients & Total patients & Without therapy $(\mathrm{n}=53)$ & With therapy $(\mathrm{n}=37)$ & Controls \\
\hline Males/Females & $41 / 49$ & $25 / 28$ & $16 / 21$ & $30 / 35$ \\
\hline Age (years) & $48 \pm 13$ & $50.21 \pm 12.99$ & $49.88 \pm 13$ & $44 \pm 9$ \\
\hline $\mathrm{BMI}\left(\mathrm{Kg} / \mathrm{m}^{2}\right)$ & $28 \pm 5$ & $27.93 \pm 4.5$ & $27.69 \pm 5.55$ & $26 \pm 6$ \\
\hline Smokers & 24 & 13 & 11 & 18 \\
\hline SACE (U/L) & $44 \pm 25$ & $46.76 \pm 25$ & $46.52 \pm 28$ & \\
\hline Brain Natriuretic Peptide (pg/dl) & $17 \pm 15$ & $19.95 \pm 22.69$ & $23.26 \pm 28.37$ & $15.8 \pm 14.3$ \\
\hline Systolic Arterial Pressure (mmHg) & $122 \pm 16$ & $126.34 \pm 16.85$ & $119.04 \pm 15.38$ & $119 \pm 17$ \\
\hline \multicolumn{5}{|l|}{ Ultrasound Parameters } \\
\hline Left Atrium (mm) & $38 \pm 5$ & $37.7 \pm 4.12$ & $38.38 \pm 4.86$ & $36 \pm 7$ \\
\hline LV End Diastolic Diameter (mm) & $50 \pm 4$ & $49.62 \pm 3.97$ & $50.31 \pm 3.24$ & $45 \pm 5$ \\
\hline Mitral E wave & $0.7 \pm 0.1$ & $0.7 \pm 0.12$ & $0.66 \pm 0.13$ & $0.9 \pm 0.2$ \\
\hline Mitral A wave & $0.67 \pm 0.1$ & $0.69 \pm 0.143$ & $0.69 \pm 0.125$ & $0.5 \pm 0.2$ \\
\hline Mitral Deceleration Time (ms) & $180 \pm 26$ & $177.09 \pm 25.51$ & $177.96 \pm 21.29$ & $185 \pm 35$ \\
\hline Mitral E/A wave & $1.08 \pm 0.32$ & $1.09 \pm 0.31$ & $1.03 \pm 0.33$ & $1.2 \pm 0.4$ \\
\hline Tricuspidal E wave & $0.5 \pm 0.1$ & $0.5 \pm 0.08$ & $0.49 \pm 0.07$ & $0.6 \pm 0.2$ \\
\hline Tricuspidal A wave & $0.49 \pm 0.135$ & $0.51 \pm 0.147$ & $0.5 \pm 0.1$ & $0.4 \pm 0.125$ \\
\hline Tricuspidal E/A & $1.08 \pm 0.34$ & $1.08 \pm 0.35$ & $1.04 \pm 0.28$ & $1.2 \pm 0.35$ \\
\hline Tricuspidal Deceleration Time (ms) & $200 \pm 38$ & $177.09 \pm 25.51$ & $177.96 \pm 21.29$ & $195 \pm 40$ \\
\hline Ejec. Fraction (\%) & $57 \pm 5$ & $61.49 \pm 8.59$ & $62.14 \pm 7.88$ & $61 \pm 6$ \\
\hline Left Ventricular Mass (mg) & $206 \pm 56$ & $202.85 \pm 57$ & $215.6 \pm 50.21$ & $195 \pm 13$ \\
\hline \multicolumn{5}{|l|}{ Electrocardiographic Parameters } \\
\hline $\mathrm{HR}(\mathrm{bpm})$ & $76 \pm 12$ & $77.76 \pm 12.36$ & $75.96 \pm 11.43$ & $74 \pm 11$ \\
\hline Pmax (ms) & $120 \pm 14^{*}$ & $122.15 \pm 11.47^{*}$ & $116.54 \pm 15.72^{*}$ & $97 \pm 11$ \\
\hline Pmin (ms) & $76 \pm 12^{*}$ & $76.67 \pm 11.81^{*}$ & $76.06 \pm 13.16^{*}$ & $59 \pm 17$ \\
\hline Pdis (ms) & $44 \pm 13^{*}$ & $45.48 \pm 12.41^{*}$ & $40.48 \pm 12.56^{*}$ & $38 \pm 10$ \\
\hline Standard Deviation Of RR & $60 \pm 50^{*}$ & $54.93 \pm 45.27^{*}$ & $63.57 \pm 54.49^{*}$ & $90 \pm 25$ \\
\hline
\end{tabular}

"Statistical significant $(\mathrm{p}<0.05)$.

cording, the subjects remained silent and breathed freely. From each lead, the average complex was calculated by the modular ECG analysis system (MEANS). Individual average complexes were stored digitally. The mean heart rate (HR) was calculated. The standard deviation of normal R-R interval (SDNN) in the 5 minute ECG recording was used as a short-term heart rate variability index.

\subsection{P Wave Measurements}

The averaged stored ECG's of patients and controls were displayed on a high resolution computer screen. Each averaged complex in each lead was separately magnified at a magnification of $160 \mathrm{~mm} / \mathrm{s}$ and $60 \mathrm{~mm} / \mathrm{mV}$. The onset and offset of the $\mathrm{P}$ wave were defined as the junction between the $\mathrm{P}$ wave pattern and the isoelectric line and were marked with a cursor. If the baseline noise was $>10 \mu \mathrm{V}$ and/or the peak to isoelectric line $\mathrm{P}$ wave ampli- tude $<15 \mu \mathrm{V}$, the lead was excluded from the analysis. Two independent investigators measured the $\mathrm{P}$ waves without access to other information. The measurements of the two observers were averaged. Subjects with $\mathrm{P}$ waves measurable $<9$ ECG leads were excluded from the analysis.

\subsection{Definition of ECG Analysis Indices}

The following indices were derived from each measurement of each ECG:

1) The maximum $P$ wave duration in any of the measurable leads $\left(\mathrm{P}_{\max }\right)$.

2) The minimum $P$ wave duration in any of the measurable leads $\left(\mathrm{P}_{\min }\right)$.

3) $\mathrm{P}$ wave dispersion $\left(\mathrm{P}_{\mathrm{dis}}\right)$, defined as the difference between $\mathrm{P}_{\max }$ and $\mathrm{P}_{\min }$.

4) Standard Deviation of RR (SDNN), was calculated 
and used as an indirect index of autonomic nervous system.

\subsection{Accuracy of the Measurements}

Intra-observer and inter-observer mean percentage errors (absolute difference between two observations divided by the mean and expressed as a percentage for P-wave duration measurements were determined in 30 randomly selected study participants and were less than $10 \%$ in all leads.

\subsection{Statistical Analysis}

Statistical analysis was carried out with a commercially available statistical software package. Continuous data are reported as mean \pm SD. Continuous variables were normally distributed as indicated by the KolmogorovSmirnov 1-sample test except standard deviation of RR, which was log-transformed. Differences between the two groups were evaluated by using the Student's unpaired t-test for continuous and $\chi^{2}$ test for categorical variables. Bivariate correlations were calculated with Pearson's product moment method. Analysis of variance (one way ANOVA) was performed for ECG parameters among different stages of sarcoidosis and Scheffe's adjustment was performed in order to correct the significant differences among multiple comparisons. Stepwise multivariate linear regression analysis was performed to estimate independent determinants of $\mathrm{P}$ wave indices. $\mathrm{P}$ value of $<0.05$ was assumed to represent statistical significance.

\section{Results}

\subsection{Patient Characteristics}

Among 106 consecutive patients referred for possible enrollment into the study, fifty met the criteria and 16 were excluded due to arterial hypertension ( 8 patients), chronic obstructive pulmonary disease (6 patients) and cardiac arrhythmias (2 patients). The remaining 90 patients (41 males/49 females) were compared to 65 healthy volunteers (30 males/35 females). Patients' and controls' characteristics are presented in Table 1. The mean age of patients was $48 \pm 13$ years and that of the control group was $44 \pm 9$ years. The mean duration of the disease was $4.3 \pm 5.6$ years. Both the compared groups were age, sex, BMI and smoking matched (Table 1). Fifty nine patients $(65.5 \%)$ were classified as stage I, fifteen patients $(17 \%)$ stage II, five $(5.5 \%)$ at stage III while eleven $(12 \%)$ patients were at stage IV. Thirty six patients $(40 \%)$ were currently under cortisone treatment while additionally twenty six $(28.9 \%)$ had received cortisone treatment in the past (mean capture time of cortisone: $12 \pm 7$ months). The average level of SACE was within normal range while only twenty four patients
(27\%) had increased levels of SACE. Twenty four patients $(27 \%)$ were currently smokers. No significant differences were observed between sarcoidosis and control group concerning heart rate, blood pressure (Systolic and Diastolic) as well as echocardiographic indices (Table 1).

\subsection{Pulmonary Evaluation}

Pulmonary function tests showed impairment of lung function in $43(48 \%)$ patients. More specific, the averaged values (\% of the predicted values) were FEV1: $94 \pm$ 19\%, FVC: $97 \pm 19 \%$, FEV1/FVC: $92 \pm 11$, TLC $=87 \pm$ $15 \%$ and DLCO $=85 \pm 18 \%$. Restrictive pattern (DLCO $<80 \%$, TLC $<80 \%$ ) was present in $25(29 \%)$, isolated DLCO reduction in $12(13 \%)$, obstructive pattern $(\mathrm{FEV} / \mathrm{FVC}<70 \%$ ) and mixed form (DLCO $<80 \%$, TLC $<80 \%, \mathrm{FEV} / \mathrm{FVC}<70 \%)$ in $6(6.6 \%)$ patients.

\section{3. $P$ Wave Indices}

Measurements of maximum, minimum and dispersion of $\mathrm{P}$ wave in the two study groups are presented in Table 1. More specific, $\mathrm{P}$ maximum $(120 \pm 14$ vs $97 \pm 11$, p < $0.001), \mathrm{P}$ minimum $(76 \pm 12$ vs $59 \pm 171, \mathrm{p}<0.001), \mathrm{P}$ wave dispersion $(44 \pm 18$ vs $38 \pm 10, \mathrm{p}<0.001)$ and SDNN $(60 \pm 50$ vs $90 \pm 25, \mathrm{p}<0.005)$ were found to be significantly different in patient group than in controls. Further analysis of the patient group concerning $\mathrm{P}$ wave indices didn't show significant differences among the patients' groups of disease staging or the groups based on corticosteroid treatment uptake. Significant prolongation of $\mathrm{P}_{\max }(123 \pm 15 \mathrm{msec}$ vs $116 \pm 11, \mathrm{p}=0.035)$ and $\mathrm{P}_{\min }$ $(79 \pm 13$ msec vs $73 \pm 11, p<0.041)$ was seen in those patients with levels of SACE higher than $55 \mathrm{U} / \mathrm{L}$ compared to those with levels of SACE less than $54 \mathrm{U} / \mathrm{L}$. In bivariate analysis performed in patients group, ECG indices, cardiac ultrasound indices, biochemical variables and pulmonary parameters were included. Maximum $\mathrm{P}$ wave was found negatively correlated to SDNN ( $\mathrm{p}<$ $0.005, r=-0.305)$ and positively correlated to the age of the patients $(\mathrm{p}=0.042, \mathrm{r}=0.212)$, while minimum $\mathrm{P}$ wave was not related with any parameter. $\mathrm{P}$ wave dispersion was correlated positively with $\mathrm{HR}(\mathrm{p}=0.003, \mathrm{r}=$ $0.323)$ and negatively with SDNN ( $p<0.001, \mathrm{r}=$ -0.388 ). Multivariate stepwise regression analysis showed that maximum $P$ wave was independently related only with the SDNN ( $p=0.005, \beta=-0.305)$, while $\mathrm{P}$ wave dispersion was independently related with $\operatorname{HR}(\mathrm{p}=0.09$, $\beta=0.193)$ and SDNN $(\mathrm{p}=0.006, \beta=-0.318)$.

\section{Discussion}

The main finding of this study was that patients with sarcoidosis have significant higher values of maximum, minimum and dispersion of $\mathrm{P}$ wave on the surface ECG 
compared to those of healthy individuals. Also, SDNN was significant decreased in patient's group, implying a functional imbalance between sympathetic and parasympathetic system in favor of the former [23]. Of great importance is the finding that these indices did not improve with corticosteroid treatment and that they were not influenced by the stage of the disease while prolonged $\mathrm{P}$ wave was observed in patients with higher levels of SACE. P wave analysis studies have been utilized in various diseases for the assessment of arrhythmia's risk [7-12,21,24]. To our knowledge this is the first study that uses $\mathrm{P}$ wave analysis in patients with sarcoidosis.

$\mathrm{P}$ wave represents the electrical activation of both atria, which takes place sequentially from right to left atrium. $P$ wave duration is, among others, determined by the following factors: 1) Atrial conduction velocity, which is non uniform, 2) the length of the longest pathway between the right atrial site of impulse origin or sinus node and the latest area to be activated, which is typically the lateral left atrium; and 3) although not generally appreciated, both P-wave duration and morphology are not static, but dynamic, and change with shifts in the sites of the predominant atrial pacemaker [25]. The association between atrial arrhythmias and $\mathrm{P}$ wave analysis during periods of sinus rhythm is well established [7-12,21,24]. The relationship of prolonged $\mathrm{P}$ wave indices to atrial arrhythmias has clinical significance since prolonged conduction can provide an etiologic basis for the appearance of atrial arrhythmias. Turgut $e t a l$. showed that prolonged atrial conduction was a predisposing factor for the development of atrial flutter [26]. Susceptibility to this type of arrhythmias is present when there exists aberrant conduction between the atria. Prolongation of $P_{\text {dur }}$ may possibly indicate the presence of intra- or inter-atrial conduction disturbance and inhomogeneous spread can occur independently of the increase in atrial dimensions. Indeed, in this study no correlation was observed between $\mathrm{P}$ wave analysis indicis and left atrium.

Recently, $\mathrm{P}$ wave dispersion, defined as the difference between maximum and minimum $\mathrm{P}$ wave duration in 12 lead ECG, has been used to separate patients with a high risk of AF while on sinus rhythm [7,8]. Previous studies revealed the significantly higher values of $\mathrm{P}_{\max }$ and $\mathrm{P}_{\mathrm{dis}}$ in patients with paroxysmal atrial fibrillation, either idiopathic or due to hypertension compared to those of healthy subjects while another study showed that $\mathrm{P}_{\max }$ was a significant independent predictor of the recurrence of the arrhythmias [7,8,21].

Another important finding was the negative correlation of $\mathrm{P}$ wave indices with the SDNN, a marker of heart rate variability $[13,23,27]$. This relationship is in agreement with another work that showed reduced values of high frequency spectral components of heart rate variability, implying altered sympatho-vagal balance due to de- creased parasympathetic tone caused by changes of respiratory pattern in patients with sarcoidosis [13]. $\mathrm{P}$ wave duration and $\mathrm{P}$ wave dispersion have been reported to be influenced by the autonomic tone, which induces changes in the velocity of impulse propagation [28]. In addition, another study showed that increased sympathetic activity causes a significant increase in P wave dispersion. [29] As a result of these findings, the authors can suggest that the imbalance between the parasympathetic and sympathetic systems may be an underlying cause of higher values of $\mathrm{P}$ wave maximum and dispersion in sarcoidosis.

It is important to note that the authors observed prolongation of $\mathrm{P}$ wave indices in patients with increased level of SACE, implying concordance with the severity of the disease and a dynamic nature of $\mathrm{P}$ wave, which responds to fluctuations in pulmonary function tests and in the disease activity. In addition renin-angiotensinaldosterone system has been found to promote atrial fibrillation by enhancing atrial myocardial fibrosis [30]. Moreover although it is uncertain whether captopril, a well known ACE inhibitor, suppresses the production of ACE enhanced from macrophages in the granulomatous lesion, studies have demonstrated inhibition of serum ACE activity with subsequent decrease in plasma angiotensin and aldosterone, indicating that captopril may offer an effective therapeutic approach to the treatment of active stage of sarcoidosis [31].

On the other hand, Asad et al showed a rapid reversal of $\mathrm{P}$ wave characteristics responding to therapy in patients with Chronic Obstructive Lung Disease [32]. Furthermore, Carilli et al showed in a retrospective study the predictive value of $\mathrm{P}$-wave amplitude and axis in estimating the severity of nonasthmatic airway obstructive disease in the quiescent state and they demonstrated a good correlation of $\mathrm{P}$ wave amplitude and axis with FEV1/FVC and the residual volume/total lung capacity in a continuous regression equation [33]. These findings show that P-wave analysis may be used in estimating the progression of the disease severity in multiple diseases.

Identification of patients with sarcoidosis that are prone for cardiac arrhythmias is important since the prognosis of sarcoidosis is mainly determined by pulmonary and cardiac manifestations and is improved by aggressive treatment [34]. Moreover, studies have shown that survival of the patients was limited to 2 years after the development of cardiac symptoms while $67 \%$ of the patients of them died suddenly [35]. The principal histological features of heart involvement in sarcoidosis include increased fibrotic activity, lymphocyte infiltration, interstitial edema and presence of granulomas which show a localized distribution within the myocardium [36]. Postmortem studies have revealed the presence of various amounts of myocardial scar tissue, which is considered to provide the substrate for supraventricular and 
ventricular arrhythmias. Deranged microarchitecture and non-uniform anisotropic properties of the atrial myocardium may cause inhomogeneous and discontinuous propagation of sinus impulses. In addition to this effect of atrial fiber geometry on the impulse propagation, other intracellular or intercellular factors might account for the non-uniform anisotropic conduction of the atrial myocardium such as the presence of site-specific conduction delays. Expression of these pathologic findings in the ECG can be found in as many as $50 \%$ of patients even without clinical evidence of cardiac involvement, with repolarization changes, arrhythmias and conduction disturbances being the most frequent.

The main limitation of the study is the absence of follow-up. This might have been necessary in order to evaluate these indices as predictors of future arhythmic events in this setting of patients and establish their clinical significance. Also, although structural inhomogeneity in ultra-structural properties is considered to play a major role in the initiation of reentrant circuits due to the increased likelihood of unidirectional block of the premature impulse, the study showed no correlation between the atrial size and P-wave regarding the atrial structure [33]. Data regarding these indices are not available for the period before the study. These data could show this correlation.

\section{Conclusion}

Patients with sarcoidosis, in comparison to the control subjects, displayed increased values of $\mathrm{P}_{\max }, \mathrm{P}_{\min }$ and $\mathrm{P}_{\text {dis }}$ possibly reflecting an effect of the disease while an inverse correlation with SDNN was established implying an imbalance of autonomic nervous system activity. Prolongation of $\mathrm{P}_{\max }$ and $\mathrm{P}$ wave dispersion and autonomic nervous system imbalance signified increased risk for the development of atrial fibrillation, a major clinical criterion of cardiac involvement in patients with systemic sarcoidosis. This might be of importance, since identification of patients prone to develop arrhythmias played a role in the treatment and altered the course of the disease.

\section{Acknowledgements}

There was no funding or sponsorship for the design, preparation and completion of this study. There were no financial or other relationships for each author for the design, preparation and completion of this study. We have to acknowledge the assistance of Mrs Andrioti Roula, Drakopoulou Ioanna and Gialafou Irene for their assistance for the preparation of this manuscript.

\section{REFERENCES}

[1] Joint Statement of the American Thoracic Society (ATS), the European Respiratory Society (ERS) and the World Association of Sarcoidosis and Other Granulomatous Disorders (WASOG) adopted by the ATS Board of Directors and by the ERS Executive Committee, "Statement on Sarcoidosis," American Journal of Respiratory and Critical Care Medicine, Vol. 160, No. 2, 1999, pp. 736755. doi:10.1164/ajrccm.160.2.ats4-99

[2] D. Mehta, S. A. Lubitz, Z. Frankel, et al., "Cardiac Involvement in Patients with Sarcoidosis: Diagnostic and Prognostic Value of Outpatient Testing," Chest, Vol. 133, No. 6, 2008, pp. 1426-1435. doi:10.1378/chest.07-2784

[3] M. R. Patel, P. J. Cawley, J. F. Heitner, et al., "Detection of Myocardial Damage in Patients with Sarcoidosis," Circulation, Vol. 120, No. 20, 2009, pp. 1969-1977.

[4] A. Perry and F. Vuitch, "Causes of Death in Patients with Sarcoidosis. A Morphologic Study of 38 Autopsies with Clinicopathologic Correlations," Archives of Pathology \& Laboratory Medicine, Vol. 119, No. 2, 1995, pp. 167-172.

[5] J. C. Deng, R. P. Baughman and J. P. Lynch, "Cardiac Involvement in Sarcoidosis," Seminars in Respiratory and Critical Care Medicine, Vol. 23, No. 6, 2002, pp. 513527. doi:10.1055/s-2002-36516

[6] B. Pierre-Louis, A. Prasad and W. H. Frishman, "Cardiac Manifestations of Sarcoidosis and Therapeutic Options," Cardiology in Review, Vol. 17, No. 4, 2009, pp. 153-158. doi:10.1097/CRD.0b013e3181a1f763

[7] K. Aytemir, N. Ozer, E. Atalar, et al., "P Wave Dispersion on 12-Lead Electrocardiography in Patients with Paroxysmal Atrial Fibrillation," Pacing and Clinical Electrophysiology, Vol. 23, No. 7, 2000, pp. 1109-1112. doi:10.1111/j.1540-8159.2000.tb00910.x

[8] P. E. Dilaveris, E. J. Gialafos, S. K. Sideris, et al., "Simple Electrocardiographic Markers for the Prediction of Paroxysmal Idiopathic Atrial Fibrillation," American Heart Journal, Vol. 135, No. 5, 1998, pp. 733-738. doi:10.1016/S0002-8703(98)70030-4

[9] P. E. Dilaveris, G. K. Andrikopoulos, G. Metaxas, et al., "Effects of Ischemia on P Wave Dispersion and Maximum P Wave Duration during Spontaneous Anginal Episodes," Pacing and Clinical Electrophysiology, Vol. 22, No. 11, 1999, pp. 1640-1647. doi:10.1111/j.1540-8159.1999.tb00384.x

[10] A. Dogan, M. Ozaydin, C. Nazli, et al., "Does Impaired Left Ventricular Relaxation Affect P Wave Dispersion in Patients with Hypertension?" Annals of Noninvasive Electrocardiology, Vol. 8, No. 3, 2003, pp. 189-193. doi:10.1046/j.1542-474X.2003.08304.x

[11] F. Ozmen, E. Atalar, K. Aytemir, et al., "Effect of Balloon-Induced Acute Ischaemia on P Wave Dispersion during Percutaneous Transluminal Coronary Angioplasty," Europace, Vol. 3, No. 4, 2001, pp. 299-303. doi:10.1053/eupc.2001.0187

[12] H. Turhan, E. Yetkin, R. Atak, et al., "Increased P-Wave Duration and P-Wave Dispersion in Patients with Aortic Stenosis," Annals of Noninvasive Electrocardiology, Vol. 8, No. 1, 2003, pp. 18-21. doi:10.1046/j.1542-474X.2003.08104.x

[13] J. Sztajzel, "Heart Rate Variability: A Noninvasive Electrocardiographic Method to Measure the Autonomic Ner- 
vous System," Swiss Medical Weekly, Vol. 134, No. 3536, 2004, pp. 514-522

[14] J. Habersberger, V. Manins and A. J. Taylor, "Cardiac Sarcoidosis," Internal Medicine Journal, Vol. 38, No. 4, 2008, pp. 270-277. doi:10.1111/j.1445-5994.2007.01590.x

[15] F. Tavora, N. Cresswell, L. Li, et al., "Comparison of Necropsy Findings in Patients with Sarcoidosis Dying Suddenly from Cardiac Sarcoidosis versus Dying Suddenly from Other Causes," American Journal of Cardiology, Vol. 104, No. 4, 2009, pp. 571-577. doi:10.1016/j.amjcard.2009.03.068

[16] H. Hiraga, K. Yuwai, M. Hiroe, et al., "Guidelines for the Diagnosis of Cardiac Sarcoidosis: Study Report of Diffuse Pulmonary Diseases," Japanese Ministry of Health and Welfare, Tokyo, 1993, pp. 23-24.

[17] G. W. Hunninghake, U. Costabel, M. Ando, et al., "ATS/ ERS/WASOG Statement on Sarcoidosis," Sarcoidosis, Vasculitis and Diffuse Lung Diseases, Vol. 16, No. 2, 1999, pp. 149-173.

[18] K. Sugisaki, T. Yamaguchi, S. Nagai, et al., "Clinical Characteristics of 195 Japanese Sarcoidosis Patients Treated with Oral Corticosteroids," Sarcoidosis, Vasculitis and Diffuse Lung Diseases, Vol. 20, No. 3, 2003, pp. 222-226.

[19] M. Tahir, S. K. Sharma, S. Ashraf and H. K. Mishra, "Angiotensin Converting Enzyme Genotype Affects Development and Course of Sarcoidosis in Asian Indians," Sarcoidosis, Vasculitis and Diffuse Lung Diseases, Vol. 24, No. 2, 2007, pp. 106-112.

[20] N. B. Schiller, P. M. Shah, M. Crawford, et al., "Recommendations for Quantitation of the Left Ventricle by Two-Dimensional Echocardiography. American Society of Echocardiography Committee on Standards, Subcommittee on Quantitation of Two-Dimensional Echocardiograms," Journal of the American Society of Echocardiography, Vol. 2, No. 5, 1989, pp. 358-367.

[21] P. E. Dilaveris, E. J. Gialafos, D. Chrissos, et al., "Detection of Hypertensive Patients at Risk for Paroxysmal Atrial Fibrillation during Sinus Rhythm by Computer-Assisted P Wave Analysis," Journal of Hypertension, Vol. 17, No. 10, 1999, pp. 1463-1470. doi:10.1097/00004872-199917100-00015

[22] S. F. Nagueh, "Echocardiographic Assessment of Left Ventricular Relaxation and Cardiac Filling Pressures," Current Heart Failure Reports, Vol. 6, No. 3, 2009, pp. 154-159. doi:10.1007/s11897-009-0022-8

[23] S. Lucreziotti, A. Gavazzi, L. Scelsi, et al., "Five-Minute Recording of Heart Rate Variability in Severe Chronic Heart Failure: Correlates with Right Ventricular Function and Prognostic Implications," American Heart Journal, Vol. 139, No. 6, 2000, pp. 1088-1095. doi: $10.1067 / \mathrm{mhj} .2000 .106168$

[24] K. Senen, H. Turhan, A. Riza Erbay, et al., "P-Wave Duration and P-Wave Dispersion in Patients with Dilated Cardiomyopathy," European Journal of Heart Failure, Vol. 6, No. 5, 2004, pp. 567-569.

[25] J. Boineau, "The Prolonged P Wave and Interatrial Block. Time to Consider a Broader Concept and Different Ter- minology," Journal of Electrocardiology, Vol. 38, No. 4, 2005, pp. 327-329.

doi:10.1016/j.jelectrocard.2005.05.002

[26] O. Turgut, I. Tandogan, M. B. Yilmaz, et al., "Association of $\mathrm{P}$ Wave Duration and Dispersion with the Risk for Atrial Fibrillation: Practical Considerations in the Setting of Coronary Artery Disease," International Journal of Cardiology, Vol. 144, No. 2, 2010, pp. 322-324. doi:10.1016/j.ijcard.2009.03.023

[27] R. L. Verrier and C. Antzelevitch, "Autonomic Aspects of Arrhythmogenesis: The Enduring and the New," Current Opinion in Cardiology, Vol. 19, No. 1, 2004, pp. 2-11. doi:10.1097/00001573-200401000-00003

[28] A. N. Cheema, M. W. Ahmed, A. H. Kadish and J. J. Goldberger, "Effects of Autonomic Stimulation and Blockade on Signal-Averaged P Wave Duration," Journal of the American College of Cardiology, Vol. 26, No. 2, 1995, pp. 497-502. doi:10.1016/0735-1097(95)80028-F

[29] T. Tukek, V. Akkaya, S. Demirel, et al., "Effect of Valsalva Maneuver on Surface Electrocardiographic P-Wave Dispersion in Paroxysmal Atrial Fibrillation," American Journal of Cardiology, Vol. 85, No. 7, 2000, pp. 896-899. doi:10.1016/S0002-9149(99)00891-7

[30] R. Ramaraj, "Role of the Renin-Angiotensin System in the Promotion of Atrial Fibrillation," Acta Cardiologica, Vol. 64, No. 6, 2009, p. 843 doi:10.2143/AC.64.6.2044757

[31] K. Mizuno, M. Gotoh, J. Matsui, et al., "Acute Effects of Captopril on Serum Angiotensin-Converting Enzyme Activity, the Renin-Aldosterone System and Blood Pressure in Patients with Sarcoidosis," The Tohoku Journal of Experimental Medicine, Vol. 140, No. 1, 1983, pp. 107-108. doi:10.1620/tjem.140.107

[32] N. Asad, V. M. Johnson and D. H. Spodick, “Acute Right Atrial Strain: Regression in Normal as Well as Abnormal P-Wave Amplitudes with Treatment of Obstructive Pulmonary Disease," Chest, Vol. 124, No. 2, 2003, pp. 560564. doi:10.1378/chest.124.2.560

[33] O. A. Centurion, "Clinical Implications of the P Wave Duration and Dispersion: Relationship between Atrial Conduction Defects and Abnormally Prolonged and Fractionated Atrial Endocardial Electrograms," International Journal of Cardiology, Vol. 134, No. 1, 2009, pp. 6-8. doi:10.1016/j.ijcard.2008.12.072

[34] Y. Yazaki, M. Isobe, M. Hiroe, et al., "Prognostic Determinants of Long-Term Survival in Japanese Patients with Cardiac Sarcoidosis Treated with Prednisone," American Journal of Cardiology, Vol. 88, No. 9, 2001, pp. 10061010. doi:10.1016/S0002-9149(01)01978-6

[35] V. V. Suranagi, P. R. Malur and H. B. Bannur, "Cardiac Sarcoidosis Causing Sudden Death," Indian Journal of Pathology and Microbiology, Vol. 52, No. 4, 2009, pp. 566-567. doi: 10.4103/0377-4929.56170

[36] A. Uemura, S. Morimoto, S. Hiramitsu, et al., "Histologic Diagnostic Rate of Cardiac Sarcoidosis: Evaluation of Endomyocardial Biopsies," American Heart Journal, Vol. 138, No. 2, 1999, pp. 299-302. doi:10.1016/S0002-8703(99)70115-8 